\title{
Reflets
}

Revue ontaroise d'intervention sociale et communautaire

\section{Européennes, «actrices actives» de développement rural. Structuration de nouveaux groupes de femmes}

\section{Marie-Lise Semblat}

Volume 3, numéro 2, automne 1997

Visibles et Partenaires : Pratiques et recherches féministes

URI : https://id.erudit.org/iderudit/026175ar

DOI : https://doi.org/10.7202/026175ar

Aller au sommaire du numéro

Éditeur(s)

Reflets : Revue ontaroise d'intervention sociale et communautaire

ISSN

1203-4576 (imprimé)

1712-8498 (numérique)

Découvrir la revue

Citer cet article

Semblat, M.-L. (1997). Européennes, «actrices actives» de développement rural. Structuration de nouveaux groupes de femmes. Reflets, 3(2), 106-127.

https://doi.org/10.7202/026175ar

Tous droits réservés (C) Reflets : Revue ontaroise d'intervention sociale et communautaire, 1997
Ce document est protégé par la loi sur le droit d'auteur. L’utilisation des services d’Érudit (y compris la reproduction) est assujettie à sa politique d'utilisation que vous pouvez consulter en ligne.

https://apropos.erudit.org/fr/usagers/politique-dutilisation/ 


\section{Européennes, «actrices actives» de développement rural. Structuration de nouveaux groupes de femmes}

«Les femmes rurales ...prennent place maintenant comme «actrices actives» de transformation sociale.»

\section{Marie-Lise Semblat}

Docteur en géographie, professeur au Collège coopératif de Paris, présidente du réseau ASTER (Actrices Sociales de Territoires Européens Ruraux)

Dans le contexte de mutation actuel, le monde rural est tout à la fois lieu de contradictions et creuset d'expérimentation sociale. Des potentiels d'énergie, d'idées, d'adaptabilité permettent à des groupes sociétaux de participer au changement là même où tout paraît sans avenir. Les femmes rurales d'Europe témoignent d'émergences nouvelles possibles dans ce laboratoire. Si elles n'ont pas toujours su valoriser leurs ressources et sont restées longtemps des agents occultes du développement rural, elles prennent place maintenant comme "actrices actives» (selon leur propre expression) de transformation sociale. Alors qu'isolées, elles demeurent dans l'ombre, leurs pratiques groupales vont leur assurer la visibilité, condition indispensable de leur autonomie et de leur reconnaissance comme partenaires du développement. Des groupes de femmes, de création récente et d'un genre nouveau, se structurent en milieu rural. Expression de la diversité des composantes des populations féminines en milieu rural, ils n'ont rien à voir avec les sections féminines des syndicats ou des groupes professionnels souvent très organisés en milieu rural.

On dit le monde rural en crise, en renaissance en tout cas, en mutation; en Europe, il est considéré comme appartenant aux zones économiques périphériques qui sont marginales, mais en même temps il est valorisé et objet d'une mode. Alors qu'un 
modèle urbain s'impose à l'ensemble de la planète, le monde rural est une sorte de miroir grossissant des contradictions du monde moderne: celles qui opposent tradition et modernité, marchand et non marchand, pauvreté et consommation de masse. Lieu de nombreuses ruptures (Hervieu 1993), tant démographiques que territoriales, il est caractérisé par sa recomposition (Kayser 1990). Il est par sa complexité même, et ce malgré ses difficultés et peut-être aussi à cause d'elles, un laboratoire, un lieu de transformation. Alors que le vide s'installe et que l'espace rural se décompose, un mouvement de retour des citadins vers les campagnes commence à s'amorcer. Ces nouveaux néoruraux ont-ils compris que le retard relatif du monde rural et sa distance par rapport au modèle mondial de modernité (Chesneaux 1989) permettent de s'interroger sur d'autres choix et d'autres orientations? Il semble qu'une redécouverte du monde rural, de son potentiel d'espace, de qualité de vie, de solidarité soit en train de s'amorcer. Les travaux de Nicole Mathieu analysent ce phénomène de «retour à la terre» ${ }^{1}$ qui, après le mouvement de dépeuplement des campagnes, témoigne d'un renversement.

\section{Au coeur des paradoxes : les femmes rurales}

Les femmes se situent au coeur d'une triple révolution: des moeurs (maitrise de plus en plus généralisée de la fécondité), des emplois (passage d'une économie de produits à une économie de services offrant des chances de plus en plus grandes aux femmes) et de l'éducation. Les Européennes font partie d'un «tiercé gagnant», aux côtés des jeunes et des vieux, elles appartiennent aux groupes sociétaux «porteurs d'innovations ou de changements», mais en même temps elles restent vulnérables (Loriaux 1991: 290 ).

Des populations de plus en plus composites vivent en milieu rural et certaines y travaillent, mais les agriculteurs y sont de moins en moins nombreux. Les femmes rurales, dans leur diversité, vivent des situations particulièrement paradoxales. Elles connaissent les difficultés propres à toutes les femmes et liées aux questions de 
formation, d'emploi, de faible participation aux prises de décision. En dépit d'un lent rapprochement des modes de vie et des aspirations des urbaines et des rurales (s'exprimant par la recherche de l'indépendance, une plus grande mobilité, la progression de l'entrée des femmes dans la vie active), des difficultés spécifiques touchent les rurales d'Europe. Ces dernières sont inhérentes à la fois aux modes et conditions de vie (isolement, gestion de l'espace et difficultés de déplacements, faiblesses des équipements et infrastructures), mais aussi aux mentalités du monde rural (résistances au changement, poids des préjugés, peur de l'innovation, lenteur... ). On constate que leur recherche d'emploi, leur possibilité de promotion «se heurtent à l'étroitesse de l'offre d'emploi et aux contraintes propres aux espaces de faible densité» (SEGESA 1993).

Les constats sont unanimes en Europe même là où les traditions de solidarité sont fortes (Grèce). Il s'agit d'une «singularité des villageoises» que l'on trouve du côté de «l'isolement: difficulté du recours aux services, absence d'espace à parcourir, monotonie de la vie de la «femme au foyer» et surtout véritable servitude en cas, somme toute fréquente, d'absence de moyens de transports» (Kayser 1990: 161). «Particulièrement sensibles aux pressions de l'évolution moderne», elles subissent un décalage important entre milieu urbain et milieu rural tant «en matière de standards de vie, de possibilités d'emploi ou de formation et de contrainte sociale» (CEPFAR 1990: 337). Elles sont ainsi pour le CEPFAR (Centre européen pour la promotion et la formation en milieu rural) une

"Les rurales d'Europe sont, en dépit des dangers de marginalisation et d'exclusion, porteuses d'innovation, leurs initiatives offrant une alternative à la déprise des activités artisanales, commerciales, industrielles.» catégorie plus particulièrement menacée d'exclusion et de marginalisation.

Les travaux de sociologie rurale du CNRS (Centre national de recherche scientifique) considèrent qu'un groupe menacé de marginalisation a toujours été le moteur d'une revitalisation (jusqu'à un certain seuil, bien entendu). Les rurales d'Europe sont, en dépit des dangers de marginalisation et d'exclusion, porteuses d'innovation, leurs initiatives offrant une alternative à la déprise des activités artisanales, commerciales, industrielles. Concernées au premier chef par les carences en équipements et en services, elles exercent des pressions auprès des élus locaux, deviennent 
des forces de proposition et initient de nombreux projets. C'est ce que confirme Henri Mendras, "les groupes sociaux entreprenants se renouvellent»; après les agriculteurs, les ruraux ont pris le relais et le rôle des femmes est particulièrement valorisé. Pour le sociologue du monde rural, elles exercent une fonction d'«agent de transformation» (1986). Ces analyses, par extension, peuvent avoir une portée universelle et, au-delà des rurales d'Europe, s'appliquer aux Canadiennes francophones vivant en milieu minoritaire. En effet, ces dernières connaissent une situation tout à fait particulière liée à leur appartenance linguistique. Elles sont considérées comme doublement, voire triplement minoritaires, doublement si on envisage le fait d'être femmes et triplement si on ajoute la dimension régionale. Ceci va susciter des stratégies d'action, de défense et de changement où les combinatoires entre les formes d'exclusion peuvent être productrices de transformation sociale.

Malgré leurs difficultés, des femmes de plus en plus nombreuses cherchent à venir vivre en milieu rural; leur présence active est reconnue comme déterminante dans le maintien des activités et des populations, de l'animation et de la dynamisation des campagnes. Là où des villages sont menacés de fermetures d'écoles

«...les femmes

innovent, inventent, créent des services, diversifient les activités, des exploitations et s'organisent pour redynamiser la vie locale.» et de commerces, d'extinction des services publics, les femmes innovent, inventent, créent des services, diversifient les activités, des exploitations et s'organisent pour redynamiser la vie locale. Les néorurales (appelées blow in en Irlande) et les natives des villages ont des approches et des priorités différentes, mais elles vont oeuvrer ensemble pour le développement des services. Les premières cherchent à faire bénéficier au village des mêmes avantages que ceux qu'elles ont connus en ville, les secondes parce qu'elles ont particulièrement à coeur de faire vivre leur village et leur région.

Dans un milieu en mutation et en transformation, les femmes occupent une position paradoxale; leurs actions et leur rôle peuvent donc être l'objet d'une double lecture:

- selon le modèle de la croissance et de la compétitivité, les femmes continuent à être marginalisées, exclues ou menacées d'exclusion; 
- selon d'autres critères, elles peuvent, au contraire, contribuer à l'émergence de nouveaux modèles de développement.

\section{De l'invisibilité à la visibilité}

On dit les femmes traditionnellement vouées à l'écoute: «Par leur culture, elles écoutent davantage, sont plus proches des gens, mais trop écouter peut être un obstacle à leurs projets» (Women's group de Bantry, Irlande 1993). Toujours prêtes à aider, elles concourent à la réussite de nombreuses actions d'animation dans les villages par des contributions proches des activités domestiques quotidiennes (réalisation de repas, garde des enfants...). Elles courent, sont écartelées entre différents lieux (réunion, travail, écoles des enfants, village, ...) et la gestion des déplacements familiaux leur revient souvent. Le dévouement est souvent au centre de leur existence; elles sont au coeur des solidarités familiales, de voisinage, présentes à la paroisse et dans les mouvements caritatifs. Ce sont elles qui animent les réseaux informels de parentèle et de proximité. C'est ainsi que les Irlandaises présentes dans les coopératives d'agro-tourisme sont considérées comme polyvalentes et devant «jongler avec leurs rôles multiples» ${ }^{2}$. Leur action au quotidien ne leur permettrait-elle donc pas de lancer des dynamiques qui facilitent leur reconnaissance?

Plusieurs expériences montrent comment des femmes, dans le cadre de projets économiques et culturels, ont pu participer au développement de l'ensemble du secteur géographique. Par leurs idées, leur énergie vitale, elles font preuve d'inventivité et d'audace, elles osent. C'est ainsi que Pauline ${ }^{3}$ en Irlande se fait traiter de crazy (folle) par son mari parce qu'elle souhaite étendre la clientèle de son tea-room (créé déjà comme complément de revenus sur la ferme et où elle sert les scones) en créant un plan d'eau qui permettrait d'attirer une fréquentation plus grande de Dublinois (Dublin est à un peu plus de $100 \mathrm{~km}$ ). Catherine, propriétaire d'une taverne à Delphes (Grèce) considère quant à elle que «la femme a beaucoup de dons, elle peut penser à beaucoup de choses 
"...l'action des

femmes en milieu rural demeure souvent sous le signe de

l'invisibilité..."
«Leurs initiatives sont

l'objet de confiscation et de détournements par les politiques et les experts, ce qui entraîne une désappropriation nuisible à leur autonomie...» en même temps", et elle ajoute: "C'est dur pour les hommes de l'accepter, ils veulent laisser les femmes dans les maisons... la femme, si elle avait la liberté, ferait beaucoup de choses, l'homme bloque la femme».

Malgré leur potentiel et leurs initiatives, l'action des femmes en milieu rural demeure souvent sous le signe de l'invisibilité; elles sont encore peu présentes dans les instances de décision des associations locales. Leurs qualités ne sont donc pas toujours valorisées, elles impulsent, incitent, lancent l'idée, voire le projet, mais n'en sont pas toujours ni les opératrices, ni les bénéficiaires. En effet, les rurales témoignent d'un manque de confiance en elles-mêmes, disent manquer de techniques d'animation de groupe, de gestion de projets et de stratégies partenariales. Elles sont très présentes dans tous les réseaux informels qui leur permettent d'agir souverainement mais sont peu identifiées dans les partenariats organisés.

Leurs projets sont très près des réalités de la vie quotidienne des villages et visent l'amélioration des conditions de vie des populations qui y vivent, mais ils restent trop souvent des microprojets, souvent très modestes, voire atypiques. Peu pris en compte par un modèle de développement qui réduit développement à progrès et croissance, ils sont renvoyés au champ de l'informel et de l'invisible. Les rurales d'Europe se retrouvent ainsi hors du coup, hors-jeu, menacées de marginalisation et d'exclusion; elles ne sont pas reconnues par les instances locales ni par les institutions. Leurs initiatives sont l'objet de confiscation et de détournements par les politiques et les experts, ce qui entraîne une désappropriation nuisible à leur autonomie et à leur possible concours au développement rural. Et pourtant leur potentiel, leurs ressources peuvent en période de crise contribuer à élaborer des réponses nouvelles aux situations de dévitalisation des territoires ruraux. Comment peut-on, alors, envisager un croisement entre les potentiels des femmes rurales et les besoins des territoires?

Ces constats ont conduit à élaborer une recherche qui, menée dans trois pays d'Europe (France, Grèce, Irlande), s'articule autour de deux questions fondamentales: 
- la position paradoxale des femmes dans le contexte des contradictions spatiales qui opposent le rural et l'urbain estelle déterminante? Comment et au bénéfice de qui?

- le croisement des identités, identité des femmes organisées en groupes et identité des territoires ruraux, peut-il être fertile? Comment peut-on rendre visible la contribution des femmes au développement et produire du changement social?

Ce sont par des pratiques inscrites dans la durée, des pratiques renouvelées et des échanges européens que notre objet de recherche a été identifié et construit dans une corrélation entre la théorie et l'action. La méthode s'est donc logiquement appuyée, d'une part sur l'observation participante, d'autre part sur l'entretien directif ou semi-directif. Les expériences ont été ici retenues dans le cadre d'une démarche relevant du vécu et de l'observé. Concrètement toutes nos observations et tous nos entretiens ont été réalisés à partir d'événements et de rencontres des divers réseaux associatifs dont nous sommes partie prenante. Nos relations avec chacun des groupes sont une construction progressive et interactive. Il ne s'agit donc pas d'une étude systématique et exhaustive, mais d'une élaboration à partir d'une méthode inductive. Cette dernière a permis de valider des hypothèses élaborées à partir de notre propre implication d'actrice de groupes de femmes en Picardie depuis 20 ans.

La recherche porte sur trois clubs de femmes grecques situés en Grèce centrale ainsi que sur une coopérative agro-touristique féminine grecque, deux women's groups irlandais du comté de Cork au sud-ouest de l'Irlande et deux groupes français, l'un en Isère et l'autre en Picardie. Les groupes de femmes irlandais coexistent dans des réseaux avec divers groupes affiliés à des fédérations dont la plus connue en milieu rural est l'ICA (Irish Countrywomen's Association - Association irlandaise des femmes rurales). Nous en avons identifié deux, au sud-ouest de l'Irlande dans le comté de Cork, à Bantry et Baltimore. En Grèce, des clubs de femmes existent dans de nombreuses localités. Certains sont nés en 1976 comme celui d'Aspra Spitia, à la fin du régime de dictature, d'autres sont apparus plus récemment comme ceux de Davlia et d'Héronia en 1994. Par leur mission polyvalente de valorisation du territoire, les coopératives agro-touristiques féminines ne se limitent pas 
aux seuls objectifs professionnels et économiques. Nous présenterons celle d'Arachova située en Grèce centrale, au pied du mont Parnasse. En France la situation est complexe, car de nombreux mouvements, fédérations et organisations initient des actions en faveur des femmes, mais ils ne semblent pas favoriser la constitution de tels groupes. Le poids des mentalités se conjugue à la pesanteur des organisations qui n'incitent pas leurs composantes féminines à s'autonomiser. Deux expériences ont été retenues parmi les nombreux groupes approchés. Il s'agit du centre social IDALOC Villages (Documenter-Informer-Animer-Lire-OrganiserCommuniquer) en Picardie et de l'association ADELE (Association des échanges locaux et européens) dans le sud des Alpes. En dépit de localisations très différentes, zone périurbaine du Vermandois proche de la ville moyenne - 62000 habitants — de SaintQuentin en Picardie et région de moyennes montagnes du Trièves, en Isère, les deux groupes sont composés de néorurales. Les premières exercent un métier en ville, les secondes ont choisi de s'installer pour vivre et travailler au village. Les groupes s'inscrivent dans des régions diversement identifiées allant de zones proches de centres urbains (Picardie, France) à des régions enclavées aux activités touristiques et sportives hivernales (ADELE dans le sud des Alpes et la coopérative agro-touristique féminine qui accueille les Athéniens venus skier sur les pistes du mont Parnasse) en passant par des zones côtières du sud-ouest irlandais.

Des structurations en groupes vont permettre aux rurales d'Europe de faire reconnaître leurs initiatives comme des contributions originales, capitales et novatrices pour concourir à l'avenir des campagnes. Les groupes identifiés participent tous à cette définition:

une unité collective réelle mais partielle, directement observable et fondée sur des attitudes collectives, continues et actives, ayant une oeuvre commune à accomplir, unité d'attitudes, d'oeuvres et de conduites qui constitue un cadre social structurable tendant vers une cohésion relative de manifestations de la sociabilité (Gurvitch 1968: 305). 
Le groupe n'est pas une simple juxtaposition d'individus, il se distingue de la foule, rassemblement fortuit d'individus, d'associations ou d'organisations vastes aux objectifs très généraux. Le groupe, contrairement à l'organisation, est caractérisé par un nombre plus limité de membres. Il est identifié alors par son système culturel, sa «mentalité» qui en fait sa «culture intériorisée», qui «porte en elle une vision du monde et génère des attitudes» (Mucchielli 1994: 22). Les femmes dispersées et inorganisées ne peuvent à elles seules susciter le changement. C'est la force groupale et la créativité portée collectivement qui vont sortir les femmes de l'ombre.

L'ensemble des groupes étudiés présentent au-delà des variables liées au contexte socio-économique, à l'histoire, aux traditions de leur pays, des caractères communs qui sont autant d'invariants propres aux groupes identifiés. Ils mettent tous en oeuvre des pratiques que nous nommons "primordiales». Pratique est pris dans le sens d' "exercice d'une activité volontaire modifiant ce qui nous entoure» et primordial ayant valeur de «ce qui est de première importance, ce qui passe ou devrait passer en première ligne» (Lalande 1960:807 et 826), ce qui parait prioritaire et fondamental.

Les constantes de ces pratiques primordiales concernent la genèse des groupes, leur double objectif, relatif à la fois à l'autonomie des femmes et à la promotion du milieu; enfin elles sont propres à la vision multidimensionnelle du monde, celle des rapports à l'espace, au temps et aux autres.

\section{Des pratiques primordiales}

"L'ensemble des pratiques des groupes traduisent ce que nous nommons pédagogie de l'action ou encore pédagogie du vivant...»

\section{Pédagogie du vivant}

L'ensemble des pratiques des groupes traduisent ce que nous nommons pédagogie de l'action ou encore pédagogie du vivant; elles rendent compte de la dynamique d'autoconstitution des groupes créés par les femmes elles-mêmes et non pas initiés du dehors. Toute circonstance est alors utilisée aux fins de la vie du groupe 
dans une construction progressive bâtie sur des rencontres, des échanges mais jamais sur des actions plaquées par opportunisme politique ou financier. La pédagogie de l'action est le contraire de l'esprit de système qui impose du dehors, applique des consignes et met en oeuvre des programmes.

Aucun des groupes ne nait donc ex nihilo, il est l'expression d'une histoire, d'une dynamique de projets et d'initiatives de femmes. La genèse d'un groupe nécessite du temps, de la maturation. D'entrée de jeu, les groupes ne proclament pas d'objectifs féministes; ceux-ci émergent à partir de conduites de projets. C'est ainsi qu'à Baltimore (village côtier du comté de Cork de quelques centaines d'habitants), le groupe est né en 1991, à partir d'une Community play school (crèche parentale) gérée par un groupe de mères qui avait suivi un "parenting course» (école des parents de douze semaines). Elles y échangeaient leurs problèmes relatifs à l'éducation des jeunes enfants; à l'issue de ce cours, de douze à quatorze mères ont souhaité poursuivre les contacts et ont été à l'origine du Women's group. À Bantry ${ }^{4}$, autre village de la côte ouest de l'Irlande du comté de Cork ${ }^{5}$, le groupe est né au sein d'un Community Resource Center (Centre de ressources communautaires) ${ }^{6}$ créé en 1991 dans le cadre d'un projet de développement communautaire, soutenu par le ministère de la Santé. C'est à l'issue d'une formation au développement personnel que l'idée de poursuivre les rencontres a vu le jour, c'est ainsi qu'est né le Bantry Women's group (groupe de femmes de Bantry).

Trois clubs de femmes ont été approchés en $\mathrm{Grèce}^{7}$, dans le département de Béotie, à Davlia, Aspra Spitia et Héronia. Ils se distinguent par leur importance numérique et leur durée d'existence. Ils ont cependant des objectifs assez semblables, liés aux réponses à trouver aux besoins locaux des femmes et de l'ensemble de la population. Les besoins recensés localement sont propres aux femmes rurales; ils concernent les carences en structures d'accueil pour les jeunes enfants, le manque de loisirs et les difficultés d'emploi des femmes (chômage ou travail saisonnier). Les problèmes d'éloignement sont considérables; le centralisme grec amène en effet les femmes à rechercher à Athènes de nombreux services médicaux, éducatifs, etc. Les clubs vont 


\section{"Les Grecques n'ont} pas seulement à lutter contre l'isolement physique, lot de nombreuses rurales $d u$ monde entier, mais aussi contre la solitude morale liée à la prégnance patriarcale dans ce pays.»

«La quête d'identité est poursuivie comme quête de sens...» tenter de résoudre ces difficultés en organisant des services ou des permanences décentralisés (ainsi celles de médecins spécialistes). Ils vont aussi satisfaire des besoins d'ordre psychologique et civique. Les Grecques n'ont pas seulement à lutter contre l'isolement physique, lot de nombreuses rurales du monde entier, mais aussi contre la solitude morale liée à la prégnance patriarcale dans ce pays.

\section{Double expression des identités}

Les pratiques groupales des rurales d'Europe les conduisent à valoriser leur expression en tant que femmes et à concourir au développement du territoire (de la localité). Ainsi les groupes d'Irlandaises disent à la fois vouloir se rencontrer, sortir de l'isolement, être plus à l'aise pour prendre la parole, conquérir l'égalité mais aussi «avoir un impact sur l'environnement», "trouver place dans la communauté» ${ }^{8}$. La recherche du développement de soi et de celui des autres sont simultanément poursuivis.

Les clubs des grecques quant à eux poursuivent des réponses adaptées aux réalités locales. Il s'agit de rompre l'isolement, «être ensemble», "sortir de la routine», mais aussi «aider les enfants», «élever le niveau intellectuel du village»". En Grèce comme en Irlande, les moyens mis en oeuvre pour réaliser les objectifs sont aussi divers que: conférences d'information, séminaires de formation, collecte de fonds pour créer des équipements, créations de services et d'animations des villages (bibliothèques, loisirs pour les jeunes, marché...).

Pour les Françaises du groupe ADELE ${ }^{10}$, ce qui les a rapprochées, c'est leur attachement au pays du Trièves et le goût de le faire vivre. Elles se retrouvent donc autour de problèmes communs liés à leur vie montagnarde. La quête d'identité est poursuivie comme quête de sens, de recherche d'aspirations à satisfaire dans un milieu où les conditions de vie sont difficiles et où des changements sont recherchés. La majorité des membres du groupe ne sont pas natives de la région, mais ont fait le choix d'y vivre, ce qui donne à la fois de la distance et un attachement. Leur choix de vivre en montagne a été guidé par la recherche de la qualité de vie; elles se disent attachées aux paysages, à la vie saine. De 
nombreux propos témoignent d'un attachement au «pays» et à sa valorisation:

Même si on ne gagne pas beaucoup d'argent on valorise le pays (Cathy).

On a quelque chose en nous qui nous pousse à vouloir un espace rural vivant (Jacqueline).

Il $y$ a une image globale entre nous de gens qui veulent tous valoriser le pays. (...) Ce qui nous unit, c'est un amour partagé pour la montagne même si la vie y est difficile. (...) Une idée commune, une identité commune (Thérèse).

On a une notion d'identité locale, de préservation de notre culture (...), on veut préserver, on ne veut pas brader (Jacqueline).

"...les groupes

francophones hors

Québec traduisent le croisement des identités de femmes et de langue. C'est cette articulation même des identités qui permet aux femmes tout à la fois de conquérir leur autonomie et de développer leurs différents milieux d'appartenance.»
Nous avons constaté une construction dynamique de l'identité ou plutôt des identités. Les groupes de femmes visent à la fois l'identité des femmes et celles des territoires où elles vivent et agissent. De la même façon, les groupes francophones hors Québec traduisent le croisement des identités de femmes et de langue. C'est cette articulation même des identités qui permet aux femmes tout à la fois de conquérir leur autonomie et de développer leurs différents milieux d'appartenance.

\section{Vision multidimensionnelle du monde}

Les pratiques des groupes nous autorisent à penser qu'elles participent à l'émergence d'une nouvelle vision du monde. Au lieu d'être univoque et linéaire, cette dernière se veut globale, multidimensionnelle. Elle est d'autant plus significative qu'elle prend place dans un monde où les idéologies, mais aussi les utopies sont en train de disparaitre. Cette vision du monde rejoint celle du développement local et se trouve en rupture avec celle de la modernité, en tout cas avec ses dérives. 
Tout en rejoignant celle du développement local, la vision des groupes la complète et l'enrichit. Les pratiques groupales des femmes rurales traduisent l'inscription territoriale des projets dans des lieux, leur construction dans la durée et la dynamique de réseau. Cette vision du monde s'oppose à celle de la modernité, relative à l'espace, au temps, et aux relations; elle éclaire la question de l'identité.

Si le développement local est un projet et une pratique, cette dernière se caractérise aussi par une autre conception de l'espace, du temps et des relations. Il s'agit de retrouver des lieux identitaires, relationnels et historiques par opposition aux «non-lieux» de la modernité (Augé 1992). Le territoire est alors centre de «prise de conscience collective d'une unité et d'une cohérence» (Pecqueur 1989: 17); le temps est ici celui de la maturation et de la construction du projet, l'opposé de l'accélération et de l'éphémère. Quant à la communication, elle ne s'exprime ni dans la hiérarchie pyramidale ni dans l'opacité extrême, elle est au contraire maillage des réseaux et fécondité des partenariats où $« 1+1=3$ » (Dommergues 1990).

Le tableau ci-dessous rend compte de ces jeux d'opposition et de rapprochement.

\section{Tableau 1}

\begin{tabular}{|c|c|c|c|}
\hline & Modernité & Développement local & Femmes \\
\hline Espace & $\begin{array}{l}\text { Déterritorialisation de la } \\
\text { production, mondialisation. } \\
\text { Espace: } \\
\text { uniformisé, éclaté, normé, } \\
\text { codé. «Surabondance } \\
\text { spatiale» (Augé). } \\
\text { «Non-lieux». }\end{array}$ & $\begin{array}{l}\text { Territoire } \\
\text { Pays: } \\
\text { communauté d'intérêts, } \\
\text { identité, cohérence, } \\
\text { conscience collective, } \\
\text { sentiment d'appartenance. }\end{array}$ & $\begin{array}{l}\text { "Faire vivre le pays et pas } \\
\text { seulement l»empêcher de } \\
\text { mourir» (ADELE) } \\
\text { Valorisation d'un pays par } \\
\text { l'agro-tourisme. }\end{array}$ \\
\hline Temps & $\begin{array}{l}\text { Temps: } \\
\text { linéaire, éphémère, } \\
\text { instantané, urgence, } \\
\text { accélération, «surabondance } \\
\text { événementielle» (Augé) }\end{array}$ & $\begin{array}{l}\text { Durée } \\
\text { Laisser le temps au temps } \\
\text { Temps du projet }\end{array}$ & $\begin{array}{l}\text { Souci d'harmonie. } \\
\text { Viser le long terme. } \\
\text { Développement durable. }\end{array}$ \\
\hline Relation & $\begin{array}{l}\text { Opacité } \\
\text { Hiérarchies pyramidales } \\
\text { Atomisation } \\
\text { "Individualisation des } \\
\text { références» (Augé) }\end{array}$ & $\begin{array}{l}\text { Dynamique d'acteurs } \\
\text { Réseaux - Partenariats } \\
\text { - Solidarités } \\
\text { Participation }\end{array}$ & $\begin{array}{l}\text { Réciprocité des échanges. } \\
\text { Recherche de l'authenticité }\end{array}$ \\
\hline
\end{tabular}


Sur les trois axes que sont l'espace, le temps et la relation se jouent donc des enjeux capitaux pour le devenir du milieu au travers de celui des femmes. C'est en termes de relations, de productions et de sources d'émancipation qu'apparaitt l'espace/temps/relationnel des pratiques des groupes.

\section{De l'espace privé à l'espace public}

"L'espace public est de plus en plus investi par les femmes qui ne se limitent pas au domaine privé ou encore qui savent en faire des passerelles vers l'univers social.»
Les groupes de femmes établissent des rapports volontaires à l'espace. Ces rapports suscitent des relations autres au temps et des formes de communication elles aussi d'une autre nature. Outre le fait que ces trois éléments structurent une vision multidimensionnelle du monde, ils concourent à la mise en mouvement des femmes. Celle-ci est spatiale, mais aussi sociale puisque les femmes conquièrent leur autonomie par les pratiques groupales de développement du milieu.

Par leurs organisations collectives territoriales, les rurales vont passer de l'espace privé et clos des maisons à l'agora, l'espace public, elles vont dépasser le territoire local du village, du canton, du comté (Irlande), du nome (Grèce), pour s'engager dans des échanges internationaux. L'espace public est de plus en plus investi par les femmes qui ne se limitent pas au domaine privé ou encore qui savent en faire des passerelles vers l'univers social. C'est le cas des coopératives agro-touristiques féminines grecques qui leur permettent d'accéder à l'autonomie. En développant des formes modernes d'organisation agricole, elles sortent de la dépendance des maris et accèdent à des revenus. Elles développent leur conscience politique par le fonctionnement démocratique de la coopérative. À partir d'activités qui leur sont familières et qui se déroulent au sein de la maison, elles vont progressivement s'approprier des lieux où les femmes occupaient précédemment peu de place. Si la conquête de l'espace fait passer du privé au public, c'est, pour le temps, la démarche inverse: elles passent d'un temps socialement organisé à du temps personnel retrouvé. Tout ceci contribue à libérer les relations avec les autres, notamment 
avec les hommes. Frosso Xaritou, présidente de la coopérative agro-touristique féminine d'Arachova n'hésite pas à dire : «nous avons un comportement plus facile dans les relations avec le village, on peut sortir, aller au café, au restaurant, discuter de nos problèmes» ${ }^{11}$.

\section{Visibles et partenaires}

"Passer du réseau naturel au partenariat nécessite pour les membres des différents groupes un détour intellectuel pour analyser les objectifs communs..."
Les femmes sont donc très présentes dans les réseaux fondés sur l'entraide et la relation où la place des sentiments est importante. Si le réseau évoque la spontanéité, l'organisation des groupes suppose la formalisation et la structuration reposant sur le contrat, la clarté, la négociation. Passer du réseau naturel au partenariat nécessite pour les membres des différents groupes un détour intellectuel pour analyser les objectifs communs, les consensus possibles avant d'envisager des modes d'organisation et de formalisation de ce partenariat. Une telle démarche s'inscrit dans une articulation de l'acteur et du système. La vie sociale se structure autour de deux pôles opposés que sont l'acteur et le système. L'un n'existe pas sans l'autre. D'une part, les groupes comme acteurs sociaux développent des jeux spécifiques et gèrent de façon propre les zones d'incertitude, en intégrant les logiques de leurs membres. D'autre part, ils prennent place au sein de l'organisation sociale dans sa globalité; leurs stratégies ne dépendent pas uniquement de leurs objectifs, mais également de «leur capacité à s'organiser pour mener une action, voire, éventuellement, à trouver leur identité pour exister de façon cohérente» (Crozier et Friedberg 1977: 215).

Les femmes rurales se sont organisées à partir d'intérêts et d'objectifs communs. L'organisation s'est structurée à partir du croisement des identités des femmes et des territoires, elle a permis de mettre en oeuvre des stratégies réconciliant l'acteur et le système. C'est par le groupe que les femmes prennent conscience des marginalisations et des exclusions, qu'elles s'organisent à la fois comme groupes de pression et comme forces de proposition. 
Les groupes décrits sont soumis à la législation en vigueur dans leur pays régissant les mouvements à but non lucratif. Ils ont déposé des statuts, se sont fait enregistrer, ont des rythmes de réunions réguliers, parfois instaurés à jour fixe et facilitent la collecte des cotisations de leurs membres. Tout en exprimant des orientations en rupture avec des pratiques et une vision du monde, ils ne sont pas pour autant subversifs. Ils s'inscrivent dans un système : ils ont, par leur existence même et leur légitimité, un sens politique, une volonté de participer à la res publica (chose publique).

Cette participation à la vie publique et collective se traduit par l'établissement de partenariats denses. Tous les clubs grecs ont des contacts avec la mairie, avec les partenaires institutionnels locaux (préfecture, représentants du ministère de l'Agriculture...), avec les autres clubs du village; ils ont aussi des contacts entre eux. Les Irlandaises de Baltimore se battent pour obtenir un Community Hall (une maison des associations), participent à la rédaction du bulletin local. Elles collaborent avec un mouvement de la ville proche de Cork.

Les activités économiques et/ou culturelles des groupes les mettent en relation avec la collectivité dans son ensemble, que ce soit les coopératives agro-touristiques grecques, ADELE (par leurs activités d'accueil touristique) ou encore le groupe de femmes de DIALOC-Villages par la dynamique créée par le Festival de

«Les groupes de femmes en tant qu'acteurs collectifs de la vie sociale ne sont donc pas coupés $d u$ système.» cinéma européen pour les femmes du monde rural. Les groupes de femmes en tant qu'acteurs collectifs de la vie sociale ne sont donc pas coupés du système. La double identité de territoire et de femmes légitime le groupe lui-même dans son existence et ses finalités. La vie relationnelle est un élément important de la vie des groupes, elle est une composante des éléments culturels qui permettent la constitution du groupe, mais aussi ses capacités à mobiliser, malgré les divergences voire les contradictions, des aptitudes, des connaissances. Les groupes doivent apprendre à gérer les conflits. Par l'importance accordée à la formation personnelle, ils s'en donnent les moyens, ils réalisent la mise en concordance des intérêts et des énergies. Leur cohésion et leur convergence internes ne sont pas antinomiques d'une stratégie 
"Au jour le jour, les femmes déploient des stratégies d'acteurs en lien avec le système, mais aussi en rupture avec une logique et des visions du monde portées et véhiculées par le système.»

"Les groupes oscillent en permanence entre la lutte contre la marginalisation et la recherche d'innovation.» partenariale, c'est tout au contraire le consensus du groupe qui permet de créer des synergies avec les partenaires locaux.

Les pratiques sont des pratiques paradoxales. Au jour le jour, les femmes déploient des stratégies d'acteurs en lien avec le système, mais aussi en rupture avec une logique et des visions du monde portées et véhiculées par le système. L'expression de rupture s'exprime par rapport à des convictions relatives à des valeurs non partagées, mais nullement par rapport à la structure sociale en tant que telle. Les groupes cherchent par des partenariats et des stratégies, à travailler de façon inclusive et non exclusive, à coopérer plutôt qu'à entrer en compétition. Il s'agit toujours plus de mettre ensemble et d'englober que d'affronter et de mettre en oeuvre des conduites agressives.

Les groupes sont structurés et organisés, c'est un gage de leur réussite; ils se veulent des acteurs constructifs de la vie sociale, la notion d'empowerment fréquemment utilisée outre-Atlantique ne voulant pas dire gagner en pouvoir, mais plutôt gagner en autonomie et en affirmation de soi. C'est, comme on l'a vu, la mise en oeuvre du projet qui est prioritaire, qui permet cette affirmation, exprime le souci qu'ont les femmes de participer et de construire, non d'entrer en compétition.

Structuration et organisation des groupes ne sont pas synonymes de luttes de pouvoir, ne figent pas les groupes dans des cadres rigides, mais elles se montrent compatibles avec des démarches de changement. Les pratiques des groupes expriment des tensions qui, au lieu d'exclure (rupture) ou d'inclure (intégration), structurent et ordonnent l'institution. Il ne s'agit en aucun cas d'organiser la subversion; il ne s'agit pas davantage de s'intégrer sans conditions dans les cadres institutionnels. Les groupes oscillent en permanence entre la lutte contre la marginalisation et la recherche d'innovation. C'est l'approche de genre et l'approche territoriale qui vont paradoxalement se combiner pour produire du changement social. Les groupes sont en cela tout à fait différents des anciens mouvements d'agricultrices et de femmes rurales apparus au début du siècle comme les Cercles des fermières en Belgique et au Québec et les ICA en Irlande. 


\section{Des mouvements traditionnels aux groupes nouveaux}

"Les mouvements traditionnels sont appelés à gérer le système alors que les nouveaux groupes développent des résistances à l'égard $d u$ système.»
Les divergences entre anciens mouvements et nouveaux groupes apparaissent à la fois dans les finalités et les formes d'organisation. Les mouvements traditionnels regroupaient les agricultrices en vue de les associer au mouvement de modernisation de l'agriculture, comptant sur leur rôle d'éducatrices pour diffuser dans la famille les nouvelles valeurs. L'appel qui leur avait été lancé visait l'intégration dans une conception de l'agriculture productiviste; il s'adressait à un monde professionnel qui n'a pas toujours surmonté les dérives corporatistes. L'approche des nouveaux groupes est au contraire transversale, centrée sur le milieu et non sur une profession, c'est une approche territoriale. Les mouvements traditionnels sont appelés à gérer le système alors que les nouveaux groupes développent des résistances à l'égard du système. L'organisation est à l'image des objectifs. Les anciens mouvements combinent la structuration en pyramide, celle de leurs fédérations hiérarchisées avec celle des cercles. Ces derniers correspondent à une terminologie usitée, mais aussi à un mode de fonctionnement concentrique d'exercice du pouvoir. Les cercles organisés à la base se structurent entre eux de façon pyramidale. À l'inverse, les nouveaux groupes fonctionnent en réseau initiés par les femmes elles-mêmes; ils n'ont pas de mots d'ordre à faire passer, ils s'inscrivent davantage dans une optique de changement social que de maintien social.

L'hétérogénéité des femmes rurales à tendance à s'accroître et ne facilite pas leur identification et leur reconnaissance comme groupe social. Pour le géographe français Bernard Kayser:

Les fermmes au village, en particulier toutes celles qui ne sont pas des agricultrices, ont bien des traits communs qui pourraient suffire à caractériser un véritable groupe social; mais bien rares sont les indications selon lesquelles l'existence de ce groupe serait attestée, que ce soit par une prise de conscience ou par une stratégie (Kayser 1990: 161). 
«Par leur organisation collective, les rurales expriment les prises de conscience et les stratégies permettant de les identifier comme groupe social.»
Une approche territoriale globale se substituant à l'approche exclusivement professionnelle de l'univers agricole va contribuer au décloisonnement et faciliter des alliances. Par leur organisation collective, les rurales expriment les prises de conscience et les stratégies permettant de les identifier comme groupe social. À partir des groupes, les femmes acquièrent la capacité d'élaborer des projets et revendiquent la priorité du projet sur le pouvoir, ce qui conduit à considérer le projet au service du pouvoir et non comme une fin en soi. C'est ainsi que les membres des groupes s'engageront dans les instances de décisions (au niveau municipal, régional...) pour consolider et pérenniser leurs projets, en éviter les dérives et les confiscations; c'est alors que d'incitatrices des projets, les rurales d'Europe peuvent devenir de réelles opératrices de développement local.

\section{Émergence d'un féminisme territorial}

«Les femmes comme actrices des territoires ruraux introduisent la dimension de genre dans l'approche spatiale."
Les pratiques primordiales des groupes, par le croisement des identités de femmes et de territoires, conduisent à identifier l'émergence d'un «féminisme territorial», expression forgée à partir de l'appellation «syndicalisme territorial» utilisée par Félix Guattari décrivant les réalités syndicales territoriales au Chili (Guattari 1992). Le territoire est construit par le projet des acteurs organisés collectivement. Les femmes comme actrices des territoires ruraux introduisent la dimension de genre dans l'approche spatiale. Il s'agit de bien différencier le féminisme territorial du féminisme territorialisé : dans la première acception les deux termes sont complémentaires et s'enrichissent mutuellement, dans la seconde le territoire est simplement cadre et lieu d'action des groupes de femmes.

Le féminisme territorial se différencie du féminisme d'État et du féminisme institutionnel qui visent l'intégration des femmes et qui se traduisent dans les trois pays ici concernés par des mesures législatives en matière d'égalité professionnelle et de droits propres, également par la création d'instances étatiques chargées de mettre en œuvre les droits nouveaux. Les politiques d'égalité des chances 
des États et les différents programmes mis en œuvre par la Communauté européenne ont développé des moyens d'intégrer les femmes à l'Europe du marché et de pouvoir puiser dans le vivier féminin. De telles politiques ne prennent pas en compte les pratiques primordiales des groupes de femmes qui caractérisent ce que nous nommons le féminisme territorial. Expression de la société civile à l'œuvre à l'échelle territoriale, il est un concept opératoire susceptible de traduire les données nouvelles relatives à l'inscription territoriale des pratiques collectives des rurales d'Europe. S'il est réponse aux limites du féminisme institutionnel, ce féminisme territorial peut-être aussi une façon de contrer la vague d'antiféminisme et de renouveler le féminisme comme mouvement social.

\section{Bibliographie}

AUGÉ, Marc (1992). Non-lieux, Introduction à une anthropologie de la surmodernité, Paris, Seuil.

CEPFAR, (Centre européen pour la promotion et la formation en milieu agricole et rural) (1990). L'état des lieux de la ruralité: Les réalités nationales face à l'approche communautaire, Bruxelles, CEPFAR. CHESNEAUX Jean (1989). Modernité-Monde, Paris, La Découverte/Maspéro.

CROZIER, Michel et Erhard FRIEDBERG (1977). L'acteur et le système: Les contraintes de l'action collective, Paris, Seuil.

DOMMER GUES, Pierre (1990). «Les partenariats et la solidarité», Partenaires, supplément au no 7, septembre.

GUATTARI, Félix (1992). «Faillite des médias, crise de civilisation, fuite de la modernité. Pour une refondation des pratiques sociales», Le Monde diplomatique, octobre 1992, (surtitre et titre sont de la rédaction du journal, ce texte ayant été adressé par Félix Guattari au journal, quelques semaines avant son décès en août 1992).

GURVITCH, Georges (1968), La vocation actuelle de la sociologie.Vers la sociologie différentielle, $4^{e}$ édition, tome 1 ,Paris, PUF.

HERVIEU, Bertrand (1993). Les champs du futur, Paris, éditions François Bourin.

KAYSER, Bernard (1990). La renaissance rurale: sociologie des campagnes du monde occidental, Paris, Colin. LALANDE, André (1960). Vocabulaire technique et critique de la philosophie, Paris, PUF.

LORIAUX, Michel (1990). «Âge et sexe: la force des minorités», dans Gérard Mermet, Euroscopie, Les Européens: qui sont-ils? Comment vivent-ils?, Paris, Larousse, 290-291.

MATHIEU, Nicole (1995). L'emploi rural: Une vitalité cachée, Paris, L'Harmattan.

MENDRAS, Henri (1986). «Le social entraîne-t-il l'économique?» Futuribles, no 105, Association internationale Futuribles, 39-56.

MUCCHIELLI, Alex (1994). L'identité, 3e édit. rev. et augm., Paris, coll. Que sais je?, PUF. 
PECQUEUR, Bernard (1989). Le développement local, Syros, Paris.

SEGESA (Société d'études géographiques, économiques et sociologiques appliquées) (1993). Enquêtes sur trois terrains: Manche, Vosges, Loire, Paris, Ministère de l'Agriculture — DERF/SEGESA.

\section{Notes}

1. Nicole Mathieu, propos recueillis par Marie-Pierre Subtiil, Le Monde, 16 avril 1996.

2. Eve Prémachandra, rapport présenté par le groupe de pilotage de l'itinéraire irlandais, Troisième session des Universités rurales européennes, 1993.

3. Pauline et Catherine font partie des participantes de la recherche menée dans trois pays d'Europe. Nous décrivons plus loin quelques-uns des aspects méthodologique de cette recherche.

4. 2500 habitants à Bantry, district de 6392 habitants au recensement de 1991, Annual report. Community resource center (rapport annuel du Centre de ressources communautaire), Bantry 1992.

5. Le comté de Cork est l'un des 26 comtés de la République d'Irlande, il compte 412 623 habitants.

6. Assez proche des centres sociaux français.

7. La Grèce est le dixième pays à rejoindre la Communauté Européenne en 1981, avant les autres pays de l'Europe du Sud (Espagne et Portugal). La Grèce est une république parlementaire (république depuis 1973, régime parlementaire depuis la chute de la dictature en juillet 1974), c'est un pays unitaire à trois niveaux d'administration locale. On compte cinquante-sept Nomes (équivalent des départements français), circonscriptions déconcentrées de l'État et depuis peu collectivités territoriales, treize régions Périferies, entités géographiques qui n’ont aucune personnalité légale. Depuis octobre 1994 les Nomi sont administrés par des préfets (nomarchis), élus pour quatre ans. Les Nomes sont appelés à devenir des entités locales décentralisées. Seul le niveau communal est une véritable collectivité territoriale (5 562 municipalités rurales ou Kinotites et 359 municipalités urbaines, les Dimi). L'organisation territoriale de la Grèce offre de grandes similitudes avec celle de la France, mais l'administration centrale demeure encore prédominante. Si la Constitution prévoit les possibilités de coopération intercommunale, il existe encore peu de regroupements intercommunaux (300 environ).

Tout le pays est éligible pour l'objectif 1 (le développement et l'ajustement structurel des régions en retard de développement) et le développement régional grec vit plus ou moins sous la dépendance des fonds européens, notamment du FEDER (Fonds européen de développement régional) dont l'objectif est de réduire les disparités entre les régions d'Europe. 
La population agricole représente plus de $25 \%$ de la population active. Les exploitations de petite taille (4,5 ha en moyenne) sont gérées, pour les trois-quarts d'entre elles, en faire-valoir direct. Les cultures méditerranéennes de fruits, légumes et vignes dominent.

8. Entretiens avec les membres des women's groups de Bantry et Baltimore, avril 1993

9. Entretiens, avril 1994.

10. Entretiens, novembre 1993.

11. Entretien avec Frosso Xaritou, Arachova, juin 1991. 